\title{
Exploring the differences in distributional properties between Stroop and Simon effects using delta plots
}

\author{
Michael S. Pratte and Jeffrey N. Rouder \\ University of Missouri, Columbia, Missouri \\ RICHARD D. MOREY \\ University of Groningen, Groningen, The Netherlands \\ AND \\ Chuning Feng \\ University of Missouri, Columbia, Missouri
}

\begin{abstract}
Stroop and Simon tasks are logically similar and are often used to investigate cognitive control and inhibition processes. We compare the distributional properties of Stroop and Simon effects with delta plots and find different although stable patterns. Stroop effects across a variety of conditions are smallest for fast responses and increase as responses slow. Simon effects across a variety of conditions, however, are largest for fast responses but decrease, and even reverse, as responses slow. We show in three experiments that these diverging patterns hold within participants and even when the stimulus materials are identical across the tasks. These stable differences in time course serve as bedrock phenomena for building and testing theories of cognitive control and inhibition. The results of two additional experiments suggest that the determinant of time course is not simply whether the distracting information is location.
\end{abstract}

Psychologists have long known that judgments about stimuli are affected by the surrounding context. A classic context effect occurs in the Stroop task, in which a judgment about the ink color of a color term is affected by the identity of the color term (Stroop, 1935). In this case, word identity serves as task-irrelevant context information, and judgments about color are faster when color and word identity match than when they mismatch. Another context effect occurs in the Simon task (Simon, 1969), in which participants judge the color of a target square by pressing left- and right-hand response keys. Responses are faster when the stimulus is displayed on the same side as the response than when it is displayed on the opposite side. Other examples of context-effect tasks include priming tasks (Scarborough, Cortese, \& Scarborough, 1977), flanker tasks (Eriksen \& Eriksen, 1974), and identification tasks with nested letters (Navon, 1977). Because these and similar tasks can be described as assessing the effects of task-irrelevant context information on judgments about a target, we refer to them generically as context tasks (see also Kornblum \& Lee, 1995).

Context effects have been interpreted as evidence for cognitive control (Botvinick, Braver, Barch, Carter, \& Cohen, 2001), selective attention (Spieler, Balota, \& Faust, 2000), response inhibition (Ridderinkhof, 1997), and spreading activation (Neely, 1991), depending on the task and specifics of the experimental conditions. Differences in context effects across populations have been used to make claims about the effects of aging (Spieler, Balota, \& Faust, 1996), schizophrenia (Baving, Wagner, Cohen, \& Rockstroh, 2001), autism (Christ, Holt, White, \& Green, 2007), and attention deficit disorder (Ridderinkhof, Scheres, Oosterlaan, \& Sergeant, 2005) on cognitive control, selective attention, and inhibition. In fact, the robustness of the context effect across several tasks, as well as the logical similarity of these tasks, has led to a general perception that these tasks are more or less interchangeable in measuring cognitive control, selective attention, or inhibition.

In this article, we examine the time course of context effects in Stroop and Simon tasks. On the basis of the robustness of these context effects, as well as the logical similarity of the tasks, it might be expected that the time courses follow the same qualitative patterns. In fact, we report here dramatic differences. In Stroop tasks, the context effect is minimal for the fastest responses and increases as responses slow. The opposite pattern is found for Simon tasks: The context effect is maximal for the fastest responses, decreases as responses slow, and may even reverse for the slowest responses. 


\section{Distributions and Delta Plots}

The dynamics of the time course of an effect are determined by the underlying response time (RT) distributions. Figure 1A shows two hypothetical distributions for congruent and incongruent conditions. Many appropriate verbal statements may be made about this effect; for example, the mean and variance both increase with incongruency. One appealing statement from a theoretical perspective is that the effect is minimal for the fastest responses and grows as RT slows. This statement is captured by the plot in Figure 1B. This plot, the delta plot (De Jong, Liang, \& Lauber, 1994), is drawn from RT percentiles. The filled circles in Figures 1A and 1B show the 10th percentile for the distributions. The $y$-axis of the delta plot (Figure 1B) shows the difference of these percentiles, or the size of the congruency effect, at a particular percentile. In the figure, the effect is $66 \mathrm{msec}$ at the 10th percentile. The $x$-axis of the delta plot is an average of the congruent and incongruent conditions at a given percentile. For example, the average RT for the 10th percentile is $414 \mathrm{msec}$. The sets of open squares in Figures 1A and 1B show the same construction for the 65th percentile.

The remaining rows of Figure 1 show alternative distributional relationships that may underlie the congruency effect. The center row shows the case in which the slowing from incongruency is constant across the distribution. This pattern occurs if the cost of incongruency does not depend on the speed of response. As can be seen, the distributions are shifted, and the corresponding delta plot is a horizontal line with an intercept reflecting the cost. The bottom row shows two distributions that have a complex relationship. The incongruent distribution starts later than the congruent one; however, it has smaller variance. The corresponding delta plot is a negatively sloped straight line. From the delta plot, it is clear that there is a large congruency effect for the fastest responses that diminishes and even reverses as response slow (i.e., there is an advantage of incongruency for these slowest responses). Although the relationship in the bottom row seems unlikely, it is implicated in some of the data we present here.

Delta plots do not capture all of the information in the underlying RT distribution. In fact, they miss many aspects, such as the overall shapes of the distributions. They do, however, bring into sharp focus the differences among distributions, and in this regard, they are exceptionally useful. Figure 1 provides an intuitive guide about how delta plots capture relations among distributions. More formal characterizations of the relationship between delta plots and underlying distributions is provided in Speckman, Rouder, Morey, and Pratte (2008) and Zhang and Kornblum (1997).

\section{The Time Course of Effects}

In this section, we review the time course of a few previously published context-effect experiments. Before doing so, it is worthwhile to consider the time course of strength effects for comparison. Examples of strength variables include obvious manipulations, such as the intensity or duration of a to-be-detected light source. Other examples include the frequency of a word in lexical decision (Meyer,
Schvaneveldt, \& Ruddy, 1975) or even numeric distance effects (Moyer \& Landauer, 1967). Strength effects have been well studied, although typically not with delta plots. Figure 2A shows the patterns resulting from strength manipulations. The data in this case are from some of our previously published work. All of these delta plots are positive and positively increasing. This pattern implies three RT properties. First, distributions obey stochastic dominance, in that no part of the slower distribution is faster than the faster distribution. Second, the slowing increases throughout the distribution. Third, the mean and standard deviation of RT increase together. This last property is so ubiquitous that Wagenmakers and Brown (2007) have proposed, as a law, that standard deviation is proportional to mean, and they show that this relationship holds across a surprisingly wide range of strength manipulations. Consideration of these plots and of previously published RT distributions leads to the following generalization: Delta plots of strength manipulations (low strength minus high strength) start positive and increase linearly.

The delta-plot pattern in Figure 2A is concordant with many processing theories and explanations. For example, all positive and increasing delta plots are compatible with either drift rate or bound changes in the diffusion model of perception (Ratcliff, 1978), with changes in the $\tau$ parameter in the ex-Gaussian (Andrews \& Heathcote, 2001) or with the insertion of stages in processes (Ashby \& Townsend, 1980; Balota \& Chumbley, 1984). In sum, the delta plots in Figure 2A serve as a default expectation often observed and broadly consistent with several different existing theories.

Figure 2B shows delta plots from a few previously published Stroop experiments. Although there is a fair amount of variation in the methods, populations, and stimuli used, the overall pattern is clear: The Stroop manipulation acts as a strength manipulation. Delta plots are positive and increasing; that is, the effect is minimal for the fastest responses and increases thereafter. Figure $2 \mathrm{C}$ shows delta plots from two previously published studies of the Simon effect. These are surprisingly different from the Stroop and strength-effect delta plots. The Simon effect is greatest early on and decreases thereafter, and even becomes negative for the slowest responses in Burle, van den Wildenberg, and Ridderinkhof (2005). These patterns violate the Wagenmakers and Brown (2007) law that mean and standard deviation vary together. Instead, the negative delta plot slope indicates the reverse pattern: Increases in mean are accompanied by decreases in standard deviation.

The opposing patterns in the Stroop and Simon congruity effects are surprising, given the logical similarity of the tasks. At a minimum, they indicate that Stroop and Simon interference should not be considered equivalent. From a theoretical perspective, Stroop interference is broadly consistent with a host of information-processing explanations, including slower accumulation of information from lateral inhibition or the insertion of recheck stages. These explanations, however, cannot explain the negative slope in the Simon congruency effect.

A converse statement holds as well: Theories of Simon interference cannot explain Stroop interference. One expla- 
A

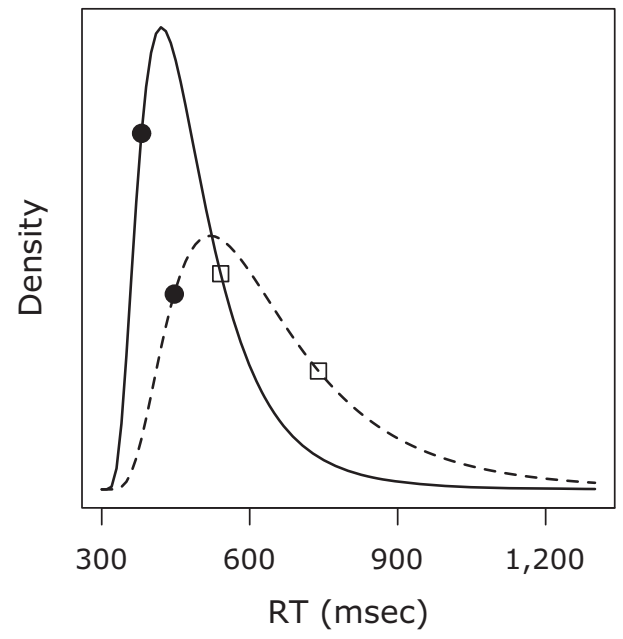

C

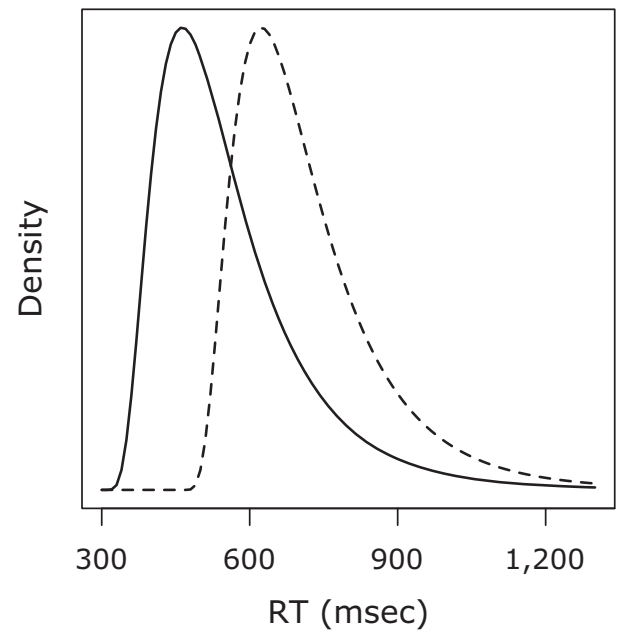

E

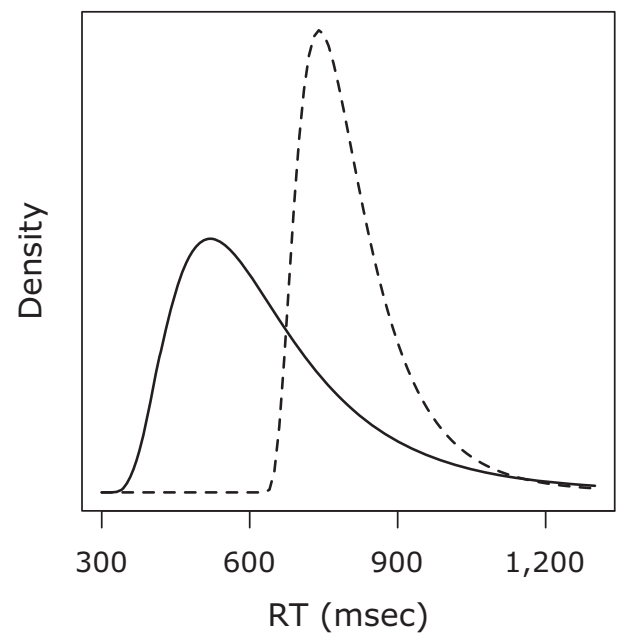

B

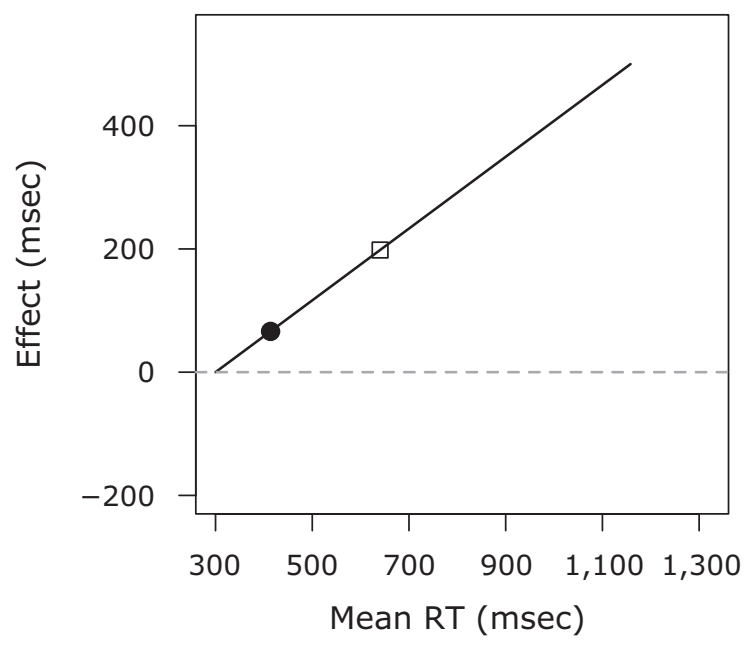

D

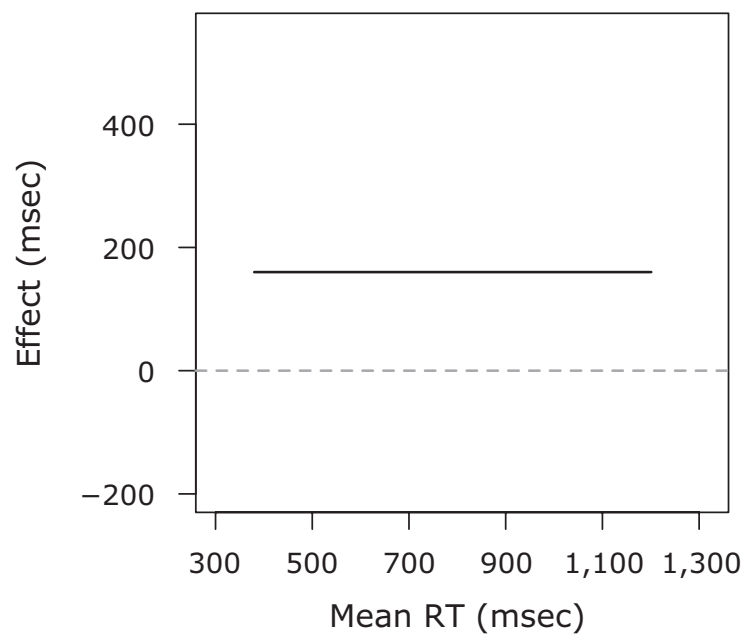

$\mathbf{F}$

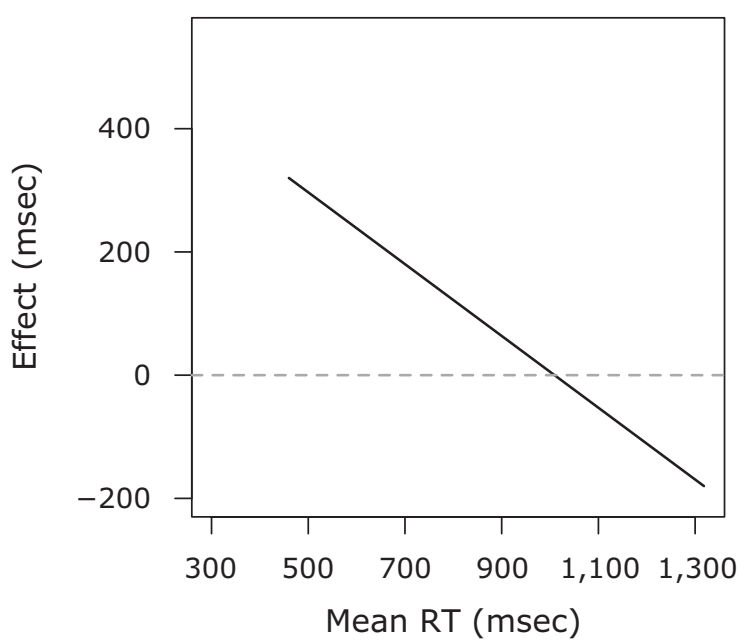

Figure 1. Distributions and delta plots. The left column shows distributions for congruent (solid) and incongruent (dashed) conditions. The right column shows the corresponding delta plots. (A and B) Congruency affects the mean and standard deviation of distributions; the resulting time course is a small effect for the quickest responses and an increasing effect thereafter. (C and D) Congruency affects only the means of distributions; the resulting time course is constant. ( $E$ and $F$ ) Congruency effects are complex (see text); the resulting time course is a large effect for the quickest responses that decreases and even reverses. 
A

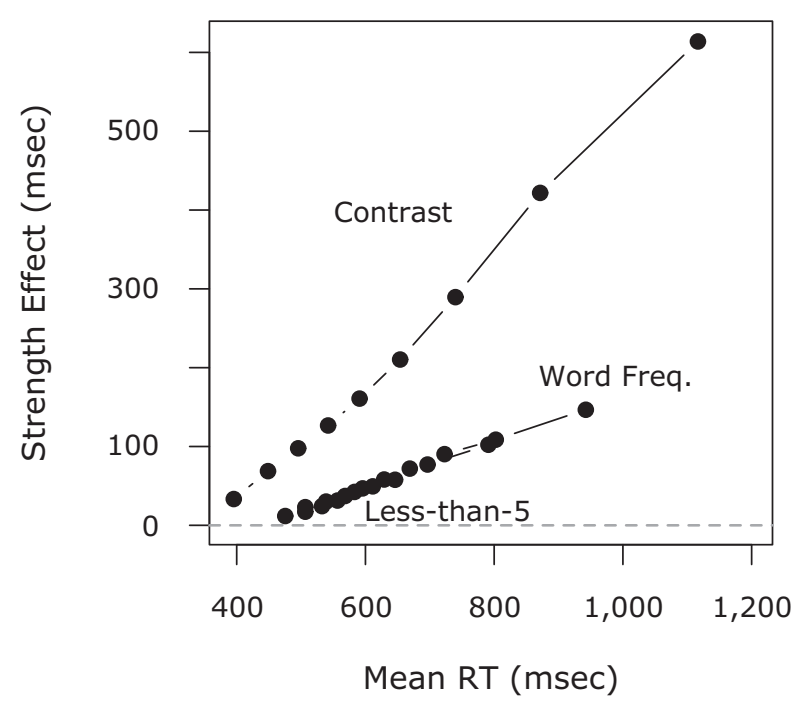

C

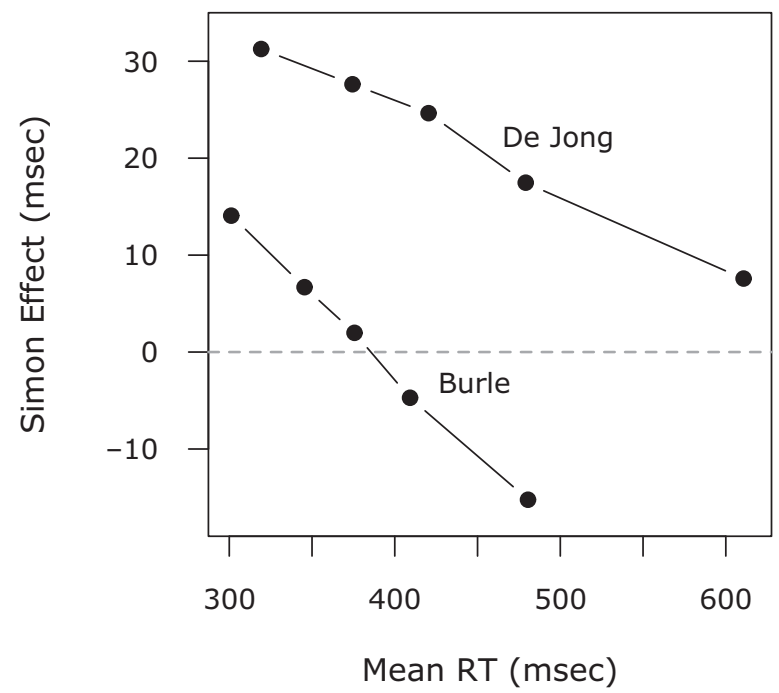

B

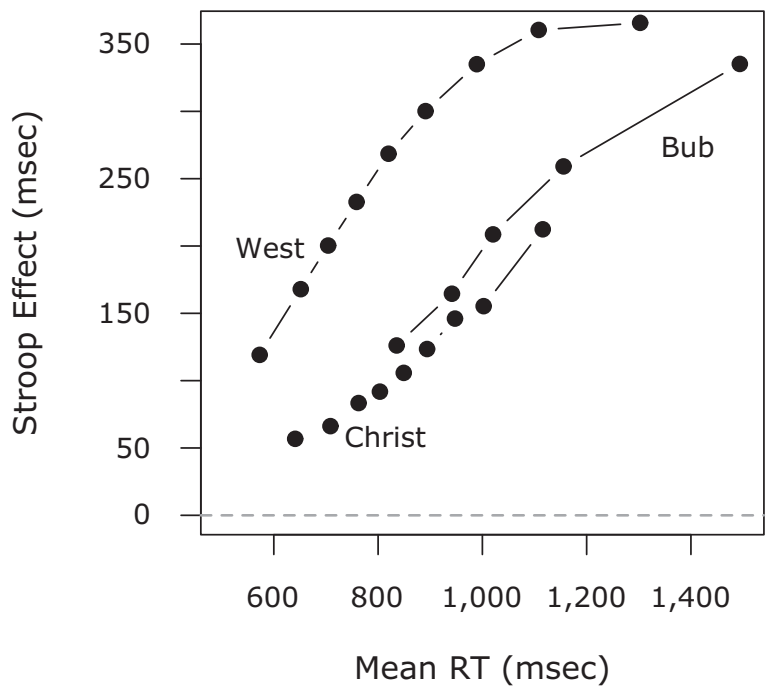

D

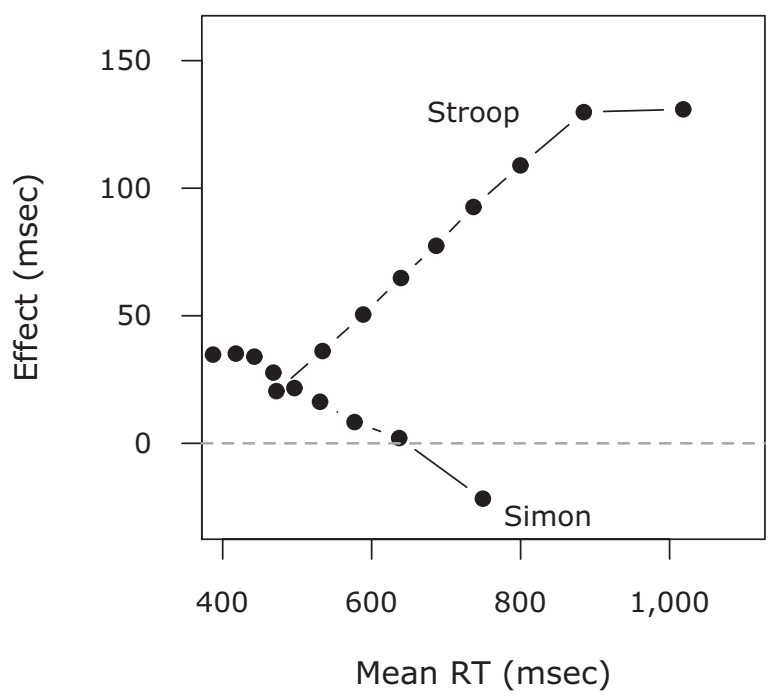

Figure 2. Delta plots of previously collected data and Experiment 1. (A) Delta plots of strength effects constructed as a contrast between a low-strength condition and a high-strength condition. The line labeled "Contrast" is from Ratcliff and Rouder (1998), Experiment 1 , in which participants judged whether a light or a dark square differed in brightness from a gray background. In the high-and low-strength conditions, squares differed markedly or subtly from the background, respectively. The line labeled "Less-than-5" is from Rouder, Lu, Speckman, Sun, and Jiang (2005), in which participants judged whether digits were less than or greater than 5. The digits 2 and 8 make up the high-strength condition; the digits 4 and 6 make up the low-strength one. The line labeled "Word Freq." is from Gomez, Ratcliff, and Perea (2007), in which participants performed a lexical decision task. Words with Kučera-Francis frequencies between 1 and 6 and between 7 and 20 made up the low-strength and high-strength conditions, respectively. (B) Delta plots of Stroop effects. The lines labeled "Christ," "West," and "Bub" are from Stroop tasks in Christ, Holt, White, and Green (2007), West (2003), and Bub, Masson, and Lalonde (2006), respectively. (C) Delta plots of Simon effects. The lines labeled "De Jong" and "Burle" are from Simon tasks in De Jong, Liang, and Lauber (1994) and Burle, van den Wildenberg, and Ridderinkhof (2005), respectively. (D) Stroop and Simon effects from Experiment 1.

nation of the negatively sloped delta plot is Ridderinkhof's (1997) two-process model. Initially, there is a quick and automatic facilitory effect of the response initiated by the context. This facilitation is then countered by inhibition of the activated response. This inhibition is slowly deployed, resulting in the observed negatively sloped time course.
Similar ideas are present in the Huber, Shiffrin, Quash, and Lyle (2002) concept of prime discounting and Eimer's (1999) model of response overcompensation. Because each of these models explains negatively sloped delta plots, however, they cannot account for the Stroop congruency effect, in which there is a positively sloped delta-plot pattern. 
The differences in time course indicate different processing explanations for Stroop and Simon effects. The evidence shown in Figures 2B and 2C, however, is metaanalytic, since it comes from contrasts across different studies, materials, labs, and participants. The goal of this article is to assess whether these time course differences hold when these elements are held constant across the tasks. In Experiment 1, we assessed whether the different delta-plot slopes hold when the same set of participants perform both tasks. Participants first performed a Simon task in which they identified the color of squares presented to the left and right sides of fixation. Then they performed a Stroop task in which they identified the color of color words. In subsequent experiments, we further equated materials across tasks.

\section{EXPERIMENT 1}

\section{Method}

Participants. Thirty-eight University of Missouri students participated in Experiment 1 in return for credit toward a course requirement.

Stimuli and Design. Stimuli in the Simon task were red and green squares subtending $2^{\circ}$ of arc and presented $4^{\circ}$ to the left or right of fixation. The Simon task was a $2 \times 2$ within-subjects balanced factorial design with stimulus color and side of presentation serving as factors. All combinations of factors occurred equally often across each block of 72 trials. Stimuli in the Stroop task were the words red, green, blue, and $X X X X$ presented in colors of red, green, and blue in the center of the screen. The Stroop task was a $4 \times 3$ within-subjects factorial design with word identity and word color serving as factors. Combinations that were congruent, incongruent, and with the neutral word $X X X X$ were presented equally often in blocks of 72 trials.

Procedure. Participants first performed seven blocks of the Simon task. Each trial began with a fixation cross presented for $700 \mathrm{msec}$ in the center of the screen. Following fixation, the target appeared and was displayed until a response was made. Following the response, a blank screen presented for $700 \mathrm{msec}$ preceded the beginning of the next trial. Participants were instructed to press the key labeled "green" (")") with the right hand if the target was green and to press the key labeled "red" ("z") with the left hand if the target was red.

Upon completing the Simon task, participants were given instructions for, and then completed, seven blocks of the Stroop task. The procedure of the Stroop task was identical to that of the Simon task, with the following exceptions. After $700 \mathrm{msec}$ of fixation, the fixation cross was replaced with a target word. Participants were instructed to press the key labeled "green" ("“/) with the right index finger if the word was colored green, to press that labeled "blue" (space) with the thumb if it was colored blue, and to press the key labeled "red" ("z") with the left index finger if it was colored red. It should be noted that whereas most researchers consider tasks in which reading interferes with color judgments as Stroop tasks, this label is not universal. Kornblum (1994), in particular, would argue that this task is not a Stroop task, because responses were made manually rather than verbally.

\section{Results}

RT served as the main dependent variable, and there was a reliable 16-msec congruency effect in the Simon task [532 vs. $548 \mathrm{msec} ; t(37)=4.36, p<.05]$ and a reliable 77-msec congruency effect in the Stroop task [681 vs. $758 \mathrm{msec} ; t(37)=12.72, p<.05]$. Additionally, although performance was highly accurate in both tasks, there were reliable congruency effects in accuracy as well [Simon, $98.5 \%$ vs. $96.9 \%, t(37)=4.15, p<.05$; Stroop, $97.2 \%$ vs. $95.0 \%, t(37)=4.14, p<.05]$, indicating that the observed RT effects do not reflect a speed-accuracy trade-off.

Delta plots of the Stroop and Simon effects are shown in Figure 2D. These plots were constructed by calculating the deciles (i.e., the 10 th, . . , 90th percentiles) for each participant in congruent and incongruent conditions for each task. The deciles were then averaged across participants. Averaging percentiles is accurate when the underlying distributions across people have the same shape and vary only in shift and scale (Jiang, Rouder, \& Speckman, 2004; Thomas \& Ross, 1980). ${ }^{1}$ For instance, all of the distributions in Figure 1 have the same shape. Rouder and Speckman (2004) showed that percentile averaging is accurate for unimodal distributions even when the same-shape assumption is modestly violated; the method is, therefore, defensible here as a means of inspecting distributional properties. Delta plots were constructed from these decile averages, as discussed previously. The trend follows the previous results: The curves are sloped positively and negatively for Stroop and Simon effects, respectively. Slopes in delta plots are ratios that describe the milliseconds of effect change for each millisecond that RT increases. For the Stroop task, the slope is .22, which indicates about a 1-msec increase in effect for every 4.5-msec increase in RT. For the Simon effect, the slope is -.16 , which indicates a $1-\mathrm{msec}$ decrease in effect for every 6-msec increase in RT.

We do not know of any statistical test of the trends in delta-plot slopes without assuming that the shape of RT distribution does not vary across conditions or people. This assumption is equivalent to modeling the underlying delta plots as straight lines. In this case, the slope reflects the ratio of standard deviations in the congruent and incongruent conditions (see Zhang \& Kornblum, 1997). This fact suggests that the ratio of standard deviations may be used to assess the statistical significance of delta-plot slopes. Alternatively, De Jong et al. (1994) fit straight lines to individuals' delta plots directly with ordinary linear regression. They then constructed contrasts across conditions on the resulting slope estimates. It would seem that contrasts on standard deviations are more appropriate than contrasts on estimated slopes, because fitting lines to delta plots violates several assumptions of ordinary least squares (OLS) regression analysis. Specifically, independence is violated, because percentiles are correlated across nearby percentiles; homogeneity of variance is violated, since the sample variance in a percentile varies across the distribution. Moreover, there is sample noise in both axes, leading to an errors-in-measurement problem (Klauer, Draine, \& Greenwald, 1998).

Given the lack of standard for assessing slope, we explored the properties of a few methods with Monte Carlo simulation. In one simulation, RT distributions were distributed as a Weibull with a shape of 1.7 ; in a second simulation, they were distributed as a log-normal with a shape of $\log \sigma=.5$. We found that the method based on OLS estimation of individuals' slopes was very reasonable (real Type I error rates ranged from .04 to .06 at the nominal .05 
level), even though the underlying assumptions were violated. Moreover, the power of this test was substantially greater than those of other methods, including contrasts on individuals' standard deviation. Hence, we use OLS regression to estimate delta plot slopes, with reasonable confidence that nominal Type I error rates are accurate. For Experiment 1, the OLS slope estimate method reveals that the slope of the Stroop effect is significantly positive $[M=.24, t(37)=7.14, p<.05]$, whereas that of the Simon effect is significantly negative $[M=-.19, t(37)=$ $7.73, p<.05]$.

\section{Discussion}

The delta-plot analyses of Experiment 1 replicate the qualitative difference in the nature of Stroop and Simon effects: The Stroop effect is small for fast responses and increases as responses slow; the Simon effect is largest for fast responses and decreases as responses slow. These diverging patterns hold when the task is manipulated within subjects.

There are several procedural differences between Stroop and Simon tasks that may account for their different patterns of effects. Among these are that the stimulus materials differ (words vs. squares) and that the number of response options differ. It is important to understand whether the delta-plot patterns reflect underlying processing differences in Stroop and Simon tasks or differences in these procedural elements. Our approach in Experiment 2 was to control these procedural differences and assess whether the delta-plot differences remained.

\section{EXPERIMENT 2}

O'Leary and Barber (1993) used a task, similar to that in Simon's (1968) early work, that is ideal for controlling procedural differences in Stroop and Simon tasks (see also Virzi \& Egeth, 1985). O'Leary and Barber used the words LEFT and RIGHT as stimuli, and these words were presented to either the left or the right of fixation. Congruent stimuli are those in which the word meaning and side of presentation match; incongruent stimuli are those in which they mismatch. There are two sets of instructions. In the Simon instruction condition, participants identify the identity of the word with the words LEFT and RIGHT mapped to left-and right-hand responses, respectively. The instructions reflect Simon interference because the incongruency occurs when the target word is presented contralateral to the correct response. In the Stroop instruction condition, participants identify the side of target presentation with the left and right side mapped to left- and right-hand responses, respectively. The instructions reflect Stroop interference because incongruency occurs when the target's meaning is in opposition with the to-be-judged feature of the target. If the delta-plot patterns show the robust differences of Experiment 1 and the previous work, these differences reflect deep processing differences rather than procedural ones. We refer to this task as the integrated Stroop-Simon task. As an aside, O'Leary and Barber performed their analysis before delta plots were introduced and did not include distributional analyses.
The integrated Stroop-Simon task is not the only way to simultaneously assess Stroop and Simon time courses. Another approach is to present colored color words (e.g., RED in green ink) on the left or right of fixation (e.g., Hommel, 1997). In this task, participants are instructed to identify the color of the word, and both Stroop and Simon effects are simultaneously present in combination. Hommel reports empirical cumulative distribution functions, from which we drew delta plots. The results showed increasing patterns for Stroop interference but flat, rather than decreasing, patterns for Simon interference.

We prefer the O'Leary and Barber (1993) integrated Stroop-Simon task to the Hommel (1997) task, for two reasons. First, Hommel's task only partially controls for stimulus materials. In the O'Leary and Barber task, the stimuli LEFT on the left and RIGHT on the right are congruent in both the Stroop and Simon tasks. Likewise, the mixed stimuli (e.g., LEFT on the right) are incongruent in both tasks. For Hommel's task, this equivalence does not hold, since the same stimulus may be congruent in Stroop color interference but incongruent in Simon location interference. Second, both Simon and Stroop interference are jointly present on each trial and need to be decomposed. This decomposition may be done by averaging, although there is no rationale for doing so.

There is one further advantage to the O'Leary and Barber (1993) integrated Stroop-Simon task: It provides a strong test of Kornblum and colleagues' (Kornblum \& Lee, 1995; Kornblum, Stevens, Whipple, \& Requin, 1999) dimensional overlap model. The model posits a taxonomy of effects depending on whether the task-relevant, distracting, or response dimensions overlap with one another. Our Stroop and Simon tasks have different levels of overlap (the Stroop and Simon tasks are Kornblum Type 3 and 4 tasks, respectively); hence, differing time courses do not provide a test of the model. In the integrated StroopSimon task, the materials and responses are equated. Consequently, both Stroop and Simon components are in the same category (Type 8; responses, distracting dimension, and task-relevant dimension all overlap). Therefore, the dimensional overlap model predicts the same time course for Stroop and Simon components. This taxonomy equivalence does not hold in the Hommel task.

\section{Method}

Participants. Thirty-eight University of Missouri students who did not participate in Experiment 1 participated in Experiment 2 in return for credit toward a course requirement.

Stimuli and Design. Stimuli consisted of the words LEFT and RIGHT presented approximately $5^{\circ}$ to the left or right of a central fixation cross. Experiment 2 was a $2 \times 2 \times 2$ within-subjects balanced factorial design with side of presentation, word identity, and task instruction serving as factors. Task instructions were held constant across a block of 72 trials. In each block, each combination of word identity and side of presentation was presented equally often.

Procedure. The procedure of Experiment 2 was identical to that of Experiment 1, with the following exceptions. Participants performed five blocks each of the Simon and Stroop tasks. All participants began with a block of the Stroop task (identify location), followed by a block of the Simon task (identify word). Task instruction proceeded to alternate between blocks for the remainder of the experiment. Before each block of Simon trials, participants were 
instructed to press the key labeled "left" ("z") with the left index finger if the word read LEFT and to press the key labeled "right" (“/”) with the right index finger if the word read RIGHT. Before each block of Stroop trials, participants were instructed to respond with the same "left" and "right" keys according to which side of the screen the word was presented on. Participants received auditory feedback on their accuracy throughout the experiment, ensuring that they continued to perform the correct task within a block.

\section{Results}

There was a reliable 28 -msec congruency effect in the Simon task [601 vs. $629 \mathrm{msec} ; t(37)=5.97, p<.05]$ and a reliable 11-msec congruency effect in the Stroop task [432 vs. $443 \mathrm{msec} ; t(37)=3.61, p<.05]$. Additionally, although performance was highly accurate in both tasks, there were reliable congruency effects in accuracy as well [Simon, 98.2\% vs. 95.6\%, t(37) = 3.69, $p<.05$; Stroop, $99.8 \%$ vs. $99.2 \%, t(37)=3.36, p<.05]$.

Delta plots for the integrated Simon and Stroop effects are shown in Figure 3B. The delta plots certainly differ from those in Experiment 1, in that the Simon effect is larger than the Stroop effect. Nonetheless, the pattern of slopes holds: The slope of the Stroop effect was significantly positive $[M=.07, t(37)=3.03, p<.05]$, whereas that of the Simon effect was significantly negative $[M=$ $-.09, t(37)=2.96, p<.05]$. These results confirm that the divergent patterns in slopes reflect processing differences in Stroop and Simon interference, rather than procedural elements.

\section{Discussion}

The typical Simon task in Experiment 1 and the Simon task in Experiment 2 in which words served as stimuli both produced negative-going delta plots, supporting previous claims that these tasks tap the same processes ( $\mathrm{Lu}$ \& Proctor, 1995). Most importantly, the diverging pattern of Stroop and Simon delta plots remains even when the materials are constant across tasks, indicating that this divergence is integral rather than a procedural artifact. We highlighted two previous theories that seemingly apply to both Stroop and Simon interference in this integrated task: Ridderinkhof and colleagues' (Ridderinkhof et al., 2005) suppression theory and Kornblum and colleagues' (Kornblum et al., 1999) dimensional overlap taxonomy. Because these theories lack a mechanism to distinguish between the Stroop and Simon interference in the integrated task, they predict that the delta plots should have similar slopes. These theories are, therefore, inconsistent with the results.

The observed Stroop effect, with an overall mean of $11 \mathrm{msec}$, is much smaller than typical Stroop effects (e.g., the 77-msec effect observed in Experiment 1). It is possible that the Stroop interference did not have time to develop, because overall responses were relatively fast in this task; if so, slowing participants may increase the size of the effect. Alternatively, the small effect may reflect an unintended task demand for the Stroop task. In the usual Stroop task, the word is presented at one location, fixation, and the participant seemingly gets a good look at it. In Experiment 2, the words were presented at varying off-center locations, and, in the Stroop task, participants simply judged the location. In this case, participants need not identify any element of the stimulus, since an assessment of a change in brightness at the periphery is sufficient. We suspected that participants simply maintained their attention at fixation and never really read the word in the integrated Stroop task. Experiment 3 was an attempt to both slow participants and force them to read the stimuli in the periphery.

\section{EXPERIMENT 3}

The key element in Experiment 3 is the addition of stop trials. Participants performed the same integrated StroopSimon task as in Experiment 2, except that, on one quarter of the trials, the word STOP was presented at one of the two lateral locations. Participants were instructed to withhold response on these stop trials and to attend carefully to the stimulus on both the Stroop and the Simon tasks. The inclusion of the stop trials meant that reading words at the periphery was task relevant. Participants not only had to take longer, but also had to direct their gaze out to the presented word.

The inclusion of stop trials did have a drawback. In some sense, the wonder of the Stroop effect is that reading is automatically engaged even when it is task irrelevant. Here, the task demand of stopping made reading the stimulus task relevant. The drawback was not critical, since the meanings of the words LEFT and RIGHT had no task relevancy for the location judgments. We acknowledge this drawback and interpret Experiment 3 as a test of the robustness of the divergent patterns. Our primary question was whether the negative delta plots would be observed for the Simon task, even when there was a large simultaneous Stroop effect.

\section{Method}

Participants. Nineteen University of Missouri students who did not participate in Experiment 1 or 2 participated in Experiment 3 in return for credit toward a course requirement.

Stimuli and Design. The stimuli and design were identical to those in Experiment 2, except that one quarter of the trials for both tasks were stop trials (presented an equal number of times at each presentation location). Task instructions for go trials were held constant across blocks of 80 trials.

Procedure. The procedure of Experiment 3 was identical to that of Experiment 2, with the following exceptions. The task that participants began with was randomized across participants (10 began with the Simon task). Before Simon blocks, participants were instructed to identify the identity of the words LEFT and RIGHT but to do nothing if the word read STOP. Before Stroop blocks, participants were instructed to identify the location of the words LEFT and RIGHT but again to do nothing if the word read STOP. For stop trials, if no response was made within a 2 -sec window, positive auditory feedback was given and the next trial followed. If participants made any response to stop trials, the text DO NOT RESPOND TO STOP!!! was displayed for $6 \mathrm{sec}$, followed by negative auditory feedback, before participants were allowed to continue with the next trial.

\section{Results}

Responses were properly withheld on $94 \%$ of the stop trials. There was a reliable $38-\mathrm{msec}$ congruency effect in the Simon task [609 vs. $647 \mathrm{msec} ; t(18)=6.43, p<.05$ ] 

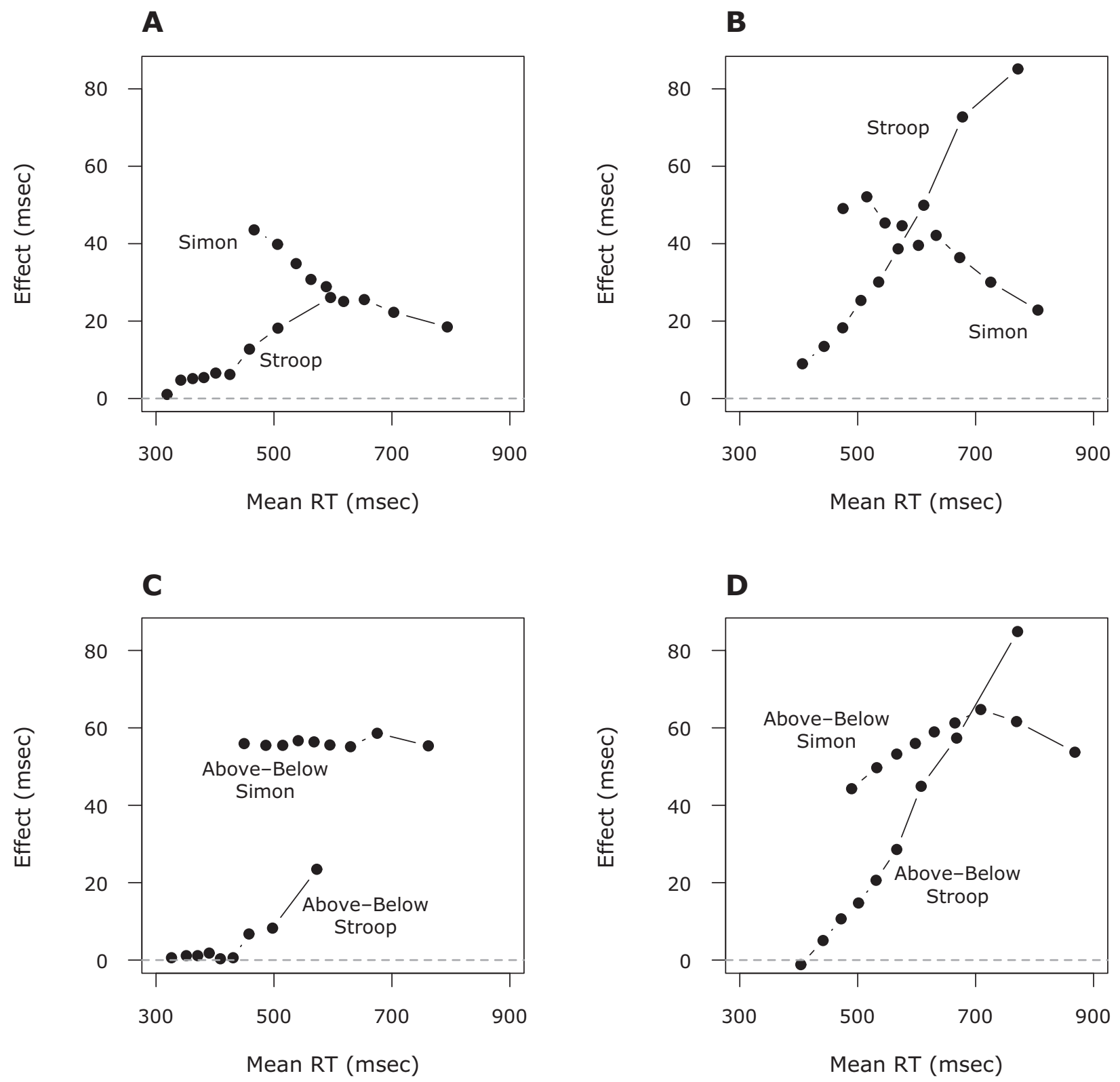

Figure 3. Delta plots of integrated Stroop and Simon effects. (A) Integrated left-right Stroop and Simon effects from Experiment 2. (B) Integrated left-right Stroop and Simon effects with stop trials from Experiment 3. (C) Integrated above-below Stroop and Simon effects from Experiment 4. (D) Integrated above-below Stroop and Simon effects with stop trials from Experiment 5.

and a reliable 39-msec congruency effect in the Stroop task [549 vs. $588 \mathrm{msec} ; t(18)=3.79, p<.05]$. Additionally, although performance was highly accurate in both tasks, there were reliable congruency effects in accuracy as well [Simon, $99.3 \%$ vs. $97.0 \%, t(18)=4.28, p<.05$; Stroop, $99.9 \%$ vs. $98.2 \%, t(18)=4.57, p<.05]$.

The delta plots for the integrated Simon and Stroop effects are shown in Figure 3B. The delta plots certainly differ from those in Experiment 2, in that the Simon and Stroop effects have nearly identical overall effect sizes. Nonetheless, the pattern of slopes holds: The slope of the Stroop effect was significantly positive $[M=.21, t(18)=$
3.96, $p<.05]$, whereas that of the Simon effect was significantly negative $[M=-.07, t(18)=2.67, p<.05]$.

\section{Discussion}

In Experiment 3, Stroop and Simon effects were fairly equivalent, in that the effect of congruency over incongruency was about the same across the tasks ( $38 \mathrm{vs} .39 \mathrm{msec}$ ). The main difference is the pattern of the delta-plot slopes, which are positive and negative for Stroop and Simon effects, respectively. Not only are there differences in slopes, the delta plots actually cross, providing strong evidence that the delta-plot slopes reflect true processing differences. 
Why are there stable differences between Stroop and Simon interference? One possibility is that the differing patterns simply reflect the fact that location and meaning information are processed differently. Accordingly, any task that has location serving as the distracting feature should have a negatively sloped delta plot, whereas any task that has meaning serving as a distracting feature should have a positively sloped delta plot. A second possibility comes from the work of Wiegand and Wascher (2005) and Vallesi, Mapelli, Schiff, Amodio, and Umiltà (2005), which implies that the processing of laterality is different from other sources of interference. Laterality may be privileged because humans have approximate leftright symmetry, which is reflected in the hemispheric organization of the brain. In the Simon task, the distracting information is the side of presentation, and the mapping from side of presentation to the response corresponds with the existing left-right symmetries. In the Stroop task, in contrast, the distracting information, although automatically and quickly processed, is semantic and may not benefit from the hemispheric organization of the brain. Given the prevailing viewpoints that Simon effects are motoric in nature (e.g., De Jong et al.,1994; Wascher, Schatz, Kuder, \& Verleger, 2001; Wiegand \& Wascher, 2005), it may be that the side of presentation affects lateralized motor activation more directly than do other sources of interference. In Experiment 4, we explored whether the negatively sloped delta plot for Simon effects results from location-based interfering information generally, or from lateralized location information specifically. We did so by adapting the integrated Stroop-Simon task to the vertical rather than to the horizontal dimension.

\section{EXPERIMENT 4}

Stimuli were the words ABOVE and BELOW presented either above or below fixation. For the Stroop task, participants identified the location of stimuli with vertically positioned buttons labeled ABOVE and BELOW, and word meaning was either congruent or incongruent with the response. For the Simon task, the same buttons were used to identify the word meaning, and the location was either congruent or incongruent with the response. Critically, the interfering location information in this above-below Simon task is not lateralized, so if the negative slope of the Simon delta plot results from lateralized location information, the abovebelow Simon delta plot should not be negative going. The stop signal was omitted from this experiment to allow for comparison with Experiment 2; Experiment 5 is the same above-below task with the stop signal included.

\section{Method}

Participants. Twenty-seven University of Missouri students who did not participate in Experiments 1-3 participated in Experiment 4 in return for credit toward a course requirement.

Stimuli and Design. The stimuli and design were identical to those in Experiment 2, except that stimuli were the words ABOVE or BELOW, and they were presented horizontally centered either above or below fixation.

Procedure. The procedure of Experiment 4 was identical to that of Experiment 2, with the following exceptions. Before Simon blocks, participants were instructed to identify the identity of the words with the keys labeled "ABOVE" ("u") and "BELOW" ("b") with their right and left index fingers, respectively. Before Stroop blocks, participants were instructed to identify the location of the words using the same keys.

\section{Results}

Although performance was highly accurate, there were reliable congruency effects in accuracy in both tasks [Simon, $98.7 \%$ vs. $94.4 \%, t(26)=4.89, p<.05$; Stroop, $99.5 \%$ vs. $99.0 \%, t(26)=3.25, p<.05]$. There was a $56-\mathrm{msec}$ congruency effect in the Simon task [563 vs. 619 msec; $t(26)=13.5, p<.05$ ] but only a small, marginally significant 7 -msec congruency effect in the Stroop task [433 vs. $441 \mathrm{msec} ; t(26)=1.87, p=.07]$.

The delta plots for the above-below Simon and Stroop effects are shown in Figure 3C. The slope of the Stroop effect was positive in magnitude but was not statistically different from zero $[M=.07, t(26)=1.42, p=.17]$. The slope of the Simon effect was also positive in magnitude but was also not significantly different from zero $[M=$ $.01, t(26)=0.33, p=.75]$.

\section{Discussion}

The vertically oriented Simon effect had a delta-plot slope of zero, whereas Simon slopes for horizontally oriented effects have negative slopes (Experiments 2 and 3). This difference can be assessed with a two-sample $t$ test on Simon slopes across Experiments 2 and $4[t(63)=2.29$, $p<.05]$. Hence, the negative-going character of the Simon delta plots is specific to horizontal lateralization. There was, however, at most, a greatly attenuated Stroop effect in Experiment 4. This attenuation was qualitatively similar to the attenuation of the Stroop effect in Experiment 2. We speculated that it may have occurred for reasons similar to those discussed previously and implemented a stop signal to force attention to the stimulus.

\section{EXPERIMENT 5}

Experiment 5 was the same vertically oriented, integrated Stroop-Simon task as that used in Experiment 4; however, a stop signal was included, as in Experiment 3.

\section{Method}

Participants. Thirty-one University of Missouri students who did not participate in Experiments 1-4 participated in Experiment 5 in return for credit toward a course requirement.

Stimuli and Design. The stimuli and design were identical to those in Experiment 4, except that the word STOP was included.

Procedure. The procedure of Experiment 5 was identical to that of Experiment 4, with the following exceptions. Before Simon blocks, participants were instructed to identify the identity of the words with the keys labeled "ABOVE" ("u") and "BELOW" ("b") with their right and left index fingers, respectively, but to do nothing if the word read sтOP ( $25 \%$ of trials). Before Stroop blocks, participants were instructed to identify the location of the words, using the same keys, but again to do nothing if the word read sTOP.

\section{Results}

Responses were properly withheld on $93 \%$ of stop trials. There was a reliable 54-msec congruency effect in the 
Simon task [633 vs. $687 \mathrm{msec} ; t(30)=9.25, p<.05]$ and a reliable $30-\mathrm{msec}$ congruency effect in the Stroop task [552 vs. $582 \mathrm{msec} ; t(30)=6.34, p<.05]$. Additionally, although performance was highly accurate in both tasks, there were reliable congruency effects in accuracy as well [Simon, $98.8 \%$ vs. $95.0 \%, t(30)=5.10, p<.05$; Stroop, $99.6 \%$ vs. $97.5 \%, t(30)=4.20, p<.05]$.

The delta plots for the above-below Simon and Stroop effects are shown in Figure 3D. The slope of the Stroop effect was significantly positive $[M=.24, t(30)=7.67$, $p<.05]$. The slope of the Simon effect was positive, but not significantly so $[M=.04, t(30)=1.59, p=.12]$.

\section{Discussion}

The vertical Stroop effect was similar in pattern to the horizontal Stroop effects in Experiments 2 and 3. This result indicates that Stroop interference is invariant to horizontal or vertical orientation, although dependent on the inclusion of a stop trial to ensure appropriate processing of stimuli. The Simon effect, however, varied across the two orientations. Simon slopes were significantly negative for the horizontal orientation (Experiments 2 and 3) but trended positive for the vertical orientation (Experiments 4 and 5). This difference can be assessed by comparing Simon slopes across Experiments 3 and 5, and the difference is indeed significant $[t(48)=2.81, p<.05]$. Hence, Experiment 5 replicates the finding from Experiment 4 , that the negative-going character of the Simon delta plots is specific to horizontal lateralization.

\section{GENERAL DISCUSSION}

In this article, we have advocated a graphical exploratory method, the delta plot, for examining time courses of context effects. When taken as a whole, the data reveal two stable and robust patterns. In Stroop tasks, congruency effects are minimal for the fastest responses and increase thereafter. In Simon tasks, in contrast, congruency effects are largest for the fastest responses, decrease as RT slows, and, in some cases, even reverse. The latter pattern is of great interest because it is in opposition to the nearly ubiquitous pattern that, across a manipulation, the standard deviation of RT increases with mean, and hence violates the law of Wagenmakers and Brown (2007). We have shown that these two patterns remain even when the tasks are performed across the same participants (Experiment 1), with the same stimuli (Experiment 2), and when the mean effects are nearly identical (Experiment 3 ). The stability of these differing patterns across our experiments, as well as others (see Figure 2), implies that they serve as a core phenomenon for theory building. Experiments 4 and 5 help to clarify the basis for the two different deltaplot patterns. In these experiments, Simon conflict from vertical orientation seems different from conflict from horizontal, lateralized orientation.

The results of Experiments 1-5 are relevant to several processing models of Stroop and Simon interference. The implications for these models are discussed in turn below.

\section{Information Accumulation Models}

In the information accumulation framework, information about a decision slowly accrues until criterion (e.g., Audley \& Pike, 1965; LaBerge, 1962; Link, 1975; Ratcliff \& Smith, 2004; Reddi \& Carpenter, 2003; Smith \& Vickers, 1988; Usher \& McClelland, 2001). There are three different loci of effects in this framework that are concordant with the positive-slope patterns observed in strength and Stroop tasks: First, information from the distracting dimension may be incorporated into the rates of accumulation (see Figure 4A). Congruent stimuli would, accordingly, have higher rates than incongruent ones. Second, information from the distracting dimension may affect the criterion (Figure 4B). For example, the semantic information in a Stroop task may come online early and affect the criterion. Although it may not be immediately obvious, changes in the criterion affect the standard deviation of the RT distribution and are, therefore, associated with a positive delta-plot slope. Likewise, if distracting information comes on early, it may be accumulated before that from the task-relevant dimension (Figure 4C). This explanation is especially appealing for priming-based tasks in which the prime precedes the target (Vorberg, Mattler, Heinecke,
A

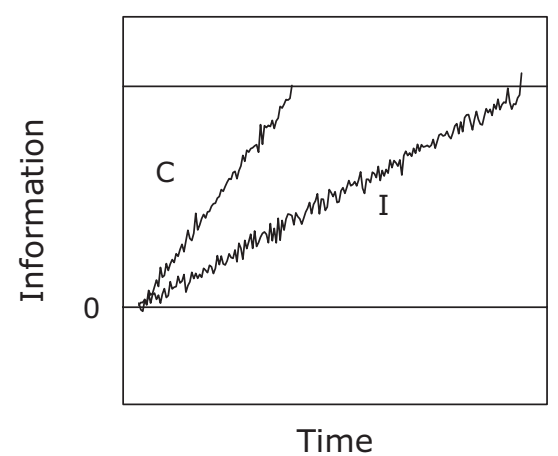

B

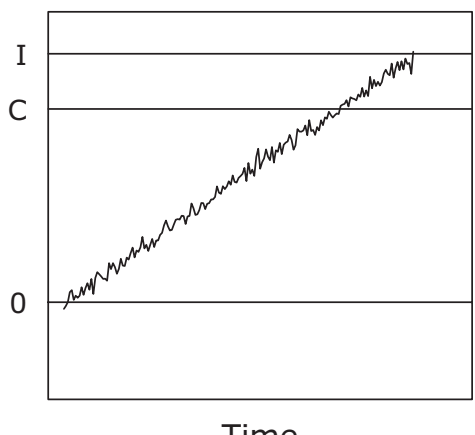

Time
C

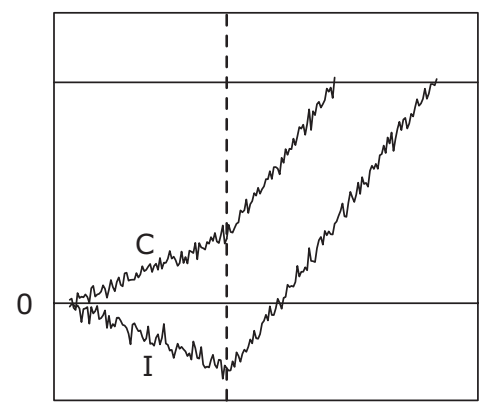

Time

Figure 4. Information accrual loci of Stroop-like context effect patterns. (A) Congruent stimuli have higher rates of information accrual than do incongruent ones. (B) Congruent stimuli have a lower decision criteria than do incongruent ones. (C) Information from irrelevant dimension accumulates before task-relevant information. 
Schmidt, \& Schwarzbach, 2003). Rouder (1996) showed that if this early accumulation is modest, it is equivalent to changing the starting point of accumulation for the taskrelevant process. The starting point is closer to criterion for congruent processes than for incongruent ones, and the result is a positively sloped delta plot. Many extant theories of Stroop interference (Cohen, Dunbar, \& McClelland, 1990; Lindsay \& Jacoby, 1994) are broadly concordant with one of these three loci and are, therefore, concordant with the Stroop-like delta plots. This broad concordance shows that delta plots provide a large-scale view of patterns, but are not suited for fine discrimination between models that make qualitatively similar predictions.

The situation is much different for the negatively sloped delta plots. These plots are fairly constraining, in that they cannot be predicted by any of the above simple explanations acting in isolation. Although one could speculate that Simon interference has two loci (e.g., rate of accumulation and criteria are affected simultaneously), such explanations strike us as post hoc and overly complex. Instead, it seems that the Simon effect simply cannot be accounted for with the broadly applicable class of information accumulation models.

\section{Translational Model}

The translation model (Virzi \& Egeth, 1985) posits that interference occurs only if the relevant information must be translated to a different modality in order to make a response. For example, in making location judgments with keypresses, both the relevant information and the keypress are spatial, so no interference is expected. In making keypress judgments to word identity, however, the verbal word information must be translated into a spatial keypress response. It is in this translation that interference occurs.

The prediction of the translational model is an asymmetry of results in the integrated Stroop and Simon tasks. Simon interference is expected, since the task-relevant information is verbal but the response is spatial. Stroop interference is not expected; both the task-relevant feature and the responses are spatial. Virzi and Egeth (1985) and O'Leary and Barber (1993) found sizable Simon effects, but only small (8- and 7-msec) and nonsignificant Stroop effects. Virzi and Egeth interpreted this pattern as support for the translational model. Our data do not support this interpretation. In Experiments 2, 3, and 5, we found a Stroop effect, even without translation. We believe that the previous failures to find Stroop interference reflect underpowered designs combined with the presence of an uninteresting task demand in which, without stop trials, participants sometimes failed to read the words at the periphery. More generally, we note that the translational model does not anticipate the two main findings herein-namely, that in lateralized tasks, Stroop and Simon interference have differing time courses, but in the vertical tasks, they have similar time courses.

\section{Horse Race Model}

The horse race model of the Stroop effect (Palef \& Olson, 1975) posits that activation of semantic and activation of color information accumulate in parallel upon stimulus presentation. This information then enters a single-channel response buffer. Semantic information, which is task irrelevant, enters more quickly than color information and, consequently, leads to interference. Conversely, color information, which is slower, does not interfere with semantic judgments.

Palef and Olson (1975) conducted an above-below experiment, as we did in Experiment 4 (i.e., with no stop trials). They found, as we did, that position affected responses to word meaning (Simon effect) but failed to find an effect of word meaning on position (no Stroop effect). This asymmetry was interpreted as evidence for the horse race model in which location information beats semantic information. Indeed, in our Experiment 4, location was identified more quickly than word meaning (Figure 3C). The results of Experiment 5, however, are problematic for this theory. Once again, location was identified more quickly than word meaning, but in this experiment, there was a large Stroop effect. More generally, we note that, like the translation model, the horse race model does not anticipate the main findings about the time course of effects.

\section{Dimensional Overlap Model}

Kornblum and colleagues' (Kornblum \& Lee, 1995; Kornblum et al., 1999) dimensional overlap model predicts a taxonomy of effects, depending on whether the task-relevant, distracting, or response dimensions overlap with one another. For example, the Stroop and Simon effects in Experiment 1 are classified as Type 4 and Type 3, respectively, so are predicted to have different patterns of effects. In both the Stroop and Simon tasks in Experiments 2 and 3, however, the task-relevant, distracting, and response dimensions all overlap with one another, such that both tasks are classified as Type 8 by the Kornblum taxonomy. These Stroop and Simon effects, however, are clearly different, as evidenced by their positive- and negative-going delta plots, respectively. Clearly, the degree of overlap between dimensions is not a critical feature for distinguishing between tasks; rather, it is whether the distracting dimension is horizontally lateralized or not that seems to differentiate Stroop and Simon interference.

\section{Activation Followed by Inhibition Accounts}

Two primary models of Simon effects have emerged. The first is the model of Ridderinkhof (1997), in which a quick, automatic congruency effect is countered by slowly evolving inhibition. In this view, the initial congruency effect for the fastest responses indexes the automatic effect; the slope of decline thereafter indexes inhibition. Although the model can explain the data patterns for Simon tasks, it does not adequately explain Stroop task patterns. A second explanation is that the Simon pattern indicates quick, automatic motor activation from the distracting information that passively decays in time. Although this explanation is appealing, it does not explain the occasional reversal of the effect for the slowest responses. These reversals can be seen unequivocally in Burle et al. (2005; see Figure 2C) and in our Experiment 1 (Figure 2D). These reversals, which indicate that the distributions for congruent and incongruent stimuli violate stochastic domi- 
nance (Speckman et al., 2008), are most concordant with Eimer's (1999) model, which posits that lateralized motoric responses may be actively inhibited. Although the Ridderinkhof and Eimer models are very similar, the appeal of the Eimer approach is that inhibition is constrained to lateralized motor activity, rather than being a general cross-task process.

\section{AUTHOR NOTE}

This research was supported by NSF Grant SES-0720229 and NIMH Grant R01-MH071418. We thank Robert West and Shawn Christ for sharing their data, and Patrick Lockwood, Robert Paterson, and Elliott Cade for helping collect the data herein. Address correspondence to M. S. Pratte, Department of Psychological Sciences, University of Missouri, 210 McAlester Hall, Columbia, MO 65211 (e-mail: prattems@) gmail.com).

\section{REFERENCES}

Andrews, S., \& HeathCote, A. (2001). Distinguishing common and task-specific processes in word identification: A matter of some moment? Journal of Experimental Psychology: Learning, Memory, \& Cognition, 27, 514-544.

Ashby, F. G., \& Townsend, J. T. (1980). Decomposing the reaction time distribution: Pure insertion and selective influences revisited. Journal of Mathematical Psychology, 21, 93-123.

Audley, R. J., \& PIKE, A. R. (1965). Some alternative stochastic models of choice. British Journal of Mathematical \& Statistical Psychology, 18, 207-225.

Balota, D. A., \& Chumbley, J. I. (1984). Are lexical decisions a good measure of lexical access? The role of word frequency in the neglected decision stage. Journal of Experimental Psychology: Human Perception \& Performance, 10, 340-357.

Baving, L., Wagner, M., Cohen, R., \& Rockstroh, B. (2001). Increased semantic and repetition priming in schizophrenic patients. Journal of Abnormal Psychology, 110, 67-75.

Botvinick, M., Braver, T., Barch, D., Carter, C., \& Cohen, J. (2001). Conflict monitoring and cognitive control. Psychological Review, 108, 624-652.

Bub, D. N., Masson, M. E. J., \& Lalonde, C. E. (2006). Cognitive control in children: Stroop interference and suppression of word reading. Psychological Science, 17, 351-357.

Burle, B., van den Wildenberg, W. P. M., \& Ridderinkhof, K. R. (2005). Dynamics of facilitation and interference in cue-priming and Simon tasks. European Journal of Cognitive Psychology, 17, 619641.

Christ, S. E., Holt, D., White, D. A., \& Green, L. (2007). Inhibitory control in children with autism. Journal of Autism \& Developmental Disorders, 37, 1155-1165.

Cohen, J., Dunbar, K., \& McClelland, J. (1990). On the control of automatic processes: A parallel distributed processing account of the Stroop effect. Psychological Review, 97, 332-361.

De Jong, R., Liang, C. C., \& Lauber, E. (1994). Conditional and unconditional automaticity: A dual-process model of effects of spatial stimulus-response concordance. Journal of Experimental Psychology: Human Perception \& Performance, 20, 731-750.

EIMER, M. (1999). Facilitatory and inhibitory effects of masked prime stimuli on motor activation and behavioural performance. Acta Psychologica, 101, 293-313.

Eriksen, B. A., \& Eriksen, C. W. (1974). Effects of noise letters upon the identification of a target letter in a nonsearch task. Perception \& Psychophysics, 16, 143-149.

Gomez, P., RatclifF, R., \& Perea, M. (2007). A model of the go/no-go task. Journal of Experimental Psychology: General, 136, 389-413.

Hommel, B. (1997). Interactions between stimulus-stimulus congruence and stimulus-response compatibility. Psychological Research, 59, 248-260.

Huber, D. E., Shiffrin, R. M., Quash, R., \& Lyle, K. B. (2002). Mechanisms of source confusion and discounting in short-term prim- ing: 1. Effects of prime duration and prime recognition. Memory \& Cognition, 30, 745-757.

Jiang, Y., Rouder, J. N., \& SPECKMAN, P. L. (2004). A note on the sampling properties of the Vincentizing (quantile averaging) procedure. Journal of Mathematical Psychology, 48, 186-195.

Klauer, K. C., Draine, S. C., \& Greenwald, A. G. (1998). An unbiased errors-in-variables approach to detecting unconscious cognition. British Journal of Mathematical \& Statistical Psychology, 51, 253-267.

Kornblum, S., \& LeE, J. (1995). Stimulus-response compatibility with relevant and irrelevant stimulus dimensions that do and do not overlap with the response. Journal of Experimental Psychology: Human Perception \& Performance, 21, 855-875.

Kornblum, S. (1994). The way irrelevant dimensions are processed depends on what they overlap with: The case of Stroop- and Simon-like stimuli. Psychological Research, 56, 130-135.

Kornblum, S., Stevens, G. T., Whipple, A., \& Requin, J. (1999). The effects of irrelevant stimuli: I. The time course of stimulus-stimulus and stimulus-response consistency effects with Stroop-like stimuli (DO Type 4 task), Simon-like tasks (DO Type 3 task), and their factorial combinations (DO Type 7 task). Journal of Experimental Psychology: Human Perception \& Performance, 25, 688-714.

LaBerge, D. (1962). A recruitment theory of simple behavior. Psychometrika, 27, 375-396.

LindSAY, D., \& JACOBY, L. (1994). Stroop process dissociations: The relationship between facilitation and interference. Journal of Experimental Psychology: Human Perception \& Performance, 20, 219-234.

LINK, S. W. (1975). The relative judgment theory of two choice response time. Journal of Mathematical Psychology, 12, 114-135.

Lu, C. H., \& Proctor, R. W. (1995). The influence of irrelevant location information on performance: A review of the Simon and spatial Stroop effects. Psychonomic Bulletin \& Review, 2, 174-207.

Meyer, D. R., Schvaneveldt, R. W., \& Ruddy, M. G. (1975). Local contextual effects on visual word recognition. In P. M. A. Rabbitt \& S. Dornic (Eds.), Attention and performance $V$ (pp. 98-118). New York: Academic Press.

Moyer, R. S., \& Landauer, T. K. (1967). Time required for judgments of numerical inequality. Nature, 215, 1519-1520.

NAVON, D. (1977). Forest before trees: The precedence of global features in visual perception. Cognition Psychology, 9, 353-383.

NeELY, J. (1991). Semantic priming effects in visual word recognition: A selective review of current findings and theories. In D. Besner \& G. Humphreys (Eds.), Basic processes in reading: Visual word recognition (pp. 264-336). Hillsdale, NJ: Erlbaum.

O'Leary, M., \& Barber, P. (1993). Interference effects in the Stroop and Simon paradigms. Journal of Experimental Psychology: Human Perception \& Performance, 19, 830-844.

Palef, S. R., \& Olson, D. R. (1975). Spatial and verbal rivalry in a Stroop-like task. Canadian Journal of Psychology, 29, 201-209.

RAtCLIFF, R. (1978). A theory of memory retrieval. Psychological Review, 85, 59-108.

Ratcliff, R., \& Rouder, J. N. (1998). Modeling response times for two-choice decisions. Psychological Science, 9, 347-356.

Ratcliff, R., \& Smith, P. L. (2004). A comparison of sequential sampling models for two-choice reaction time. Psychological Review, 111, 333-367.

Reddi, B., \& Carpenter, R. (2003). Accuracy, information and response time in a saccadic decision task. Journal of Neurophysiology, 90, 3538-3546.

RIDDERINKHOF, K. R. (1997). A dual-route processing architecture for stimulus-response correspondence effects. In B. Hommel \& W. Prinz (Eds.), Theoretical issues in $S-R$ compatibility (pp. 119-131). Amsterdam: Elsevier.

Ridderinkhof, K. R., Scheres, A., Oosterlaan, J., \& Sergeant, J. A. (2005). Distribution-analytical techniques in the study of ADAH: Delta plot analyses reveal deficits in response suppression that are eliminated by methylphenidate treatment. Journal of Abnormal Psychology, 114, 197-215.

Rouder, J. N. (1996). Premature sampling in random walks. Journal of Mathematical Psychology, 40, 289-296.

Rouder, J. N., Lu, J., SPeckman, P. L., Sun, D., \& Jiang, Y. (2005). A 
hierarchical model for estimating response time distributions. Psychonomic Bulletin \& Review, 12, 195-223.

Rouder, J. N., \& Speckman, P. L. (2004). An evaluation of the Vincentizing method of forming group-level response time distributions. Psychonomic Bulletin \& Review, 11, 419-427.

Scarborough, D. L., Cortese, C., \& Scarborough, H. S. (1977). Frequency and repetition effects in lexical memory. Journal of Experimental Psychology: Human Perception \& Performance, 3, 1-17.

SimON, J. R. (1968). Effect of ear stimulated on reaction time and movement time. Journal of Experimental Psychology, 78, 344-346.

Simon, J. R. (1969). Reactions toward the source of stimulation. Journal of Experimental Psychology, 81, 174-176.

Smith, P. L., \& Vickers, D. (1988). The accumulator model of twochoice discrimination. Journal of Mathematical Psychology, 32, 135 168 .

Speckman, P. L., Rouder, J. N., Morey, R. D., \& Pratte, M. S. (2008). Delta plots and coherent distribution ordering. American Statistician, 62, 262-266.

Spieler, D. H., Balota, D. A., \& Faust, M. E. (1996). Stroop performance in healthy younger and older adults and in individuals with dementia of the Alzheimer's type. Journal of Experimental Psychology: Human Perception \& Performance, 22, 461-479.

Spieler, D. H., Balota, D. A., \& Faust, M. E. (2000). Levels of selective attention revealed through analyses of response time distributions. Journal of Experimental Psychology: Human Perception \& Performance, 26, 506-526.

Stroop, J. R. (1935). Studies of interference in serial verbal reactions. Journal of Experimental Psychology, 18, 643-662.

Thomas, E. A. C., \& Ross, B. (1980). On appropriate procedures for combining probability distributions within the same family. Journal of Mathematical Psychology, 21, 136-152.

Usher, M., \& McClelland, J. L. (2001). On the time course of perceptual choice: The leaky competing accumulator model. Psychological Review, 108, 550-592.

Vallesi, A., Mapelli, D., Schiff, S., Amodio, P., \& Umiltà, C. (2005).
Horizontal and vertical Simon effect: Different underlying mechanisms? Cognition, 96, B33-B43.

Virzi, R. A., \& Egeth, H. E. (1985). Toward a translational model of Stroop interference. Memory \& Cognition, 13, 304-319.

Vorberg, D., Mattler, U., Heinecke, A., Schmidt, T., \& SchwarzBACH, J. (2003). Different time courses for visual perception and action priming. Proceedings of the National Academy of Sciences, 100, 6275-6280.

WAGENMAKers, E. J., \& Brown, S. (2007). On the linear relation between the mean and the standard deviation of a response time distribution. Psychological Review, 114, 830-841.

Wascher, E., Schatz, U., Kuder, T., \& Verleger, R. (2001). Validity and boundary conditions of automatic response activation in the Simon task. Journal of Experimental Psychology: Human Perception \& Performance, 27, 731-751.

WEST, R. (2003). Neural correlates of cognitive control and conflict detection in the Stroop and digit-location tasks. Neuropsychologia, 41, $1122-1135$.

Wiegand, K., \& Wascher, E. (2005). Dynamic aspects of stimulusresponse correspondence: Evidence for two mechanisms involved in the Simon effect. Journal of Experimental Psychology: Human Perception \& Performance, 31, 453-464.

Zhang, J., \& Kornblum, S. (1997). Distributional analyses and De Jong, Liang and Lauber's (1994) dual-process model of the Simon effect. Journal of Experimental Psychology: Human Perception \& Performance, 23, 1543-1551.

\section{NOTE}

1. The same-shape assumption can be formalized as follows. Let $X$ and $Y$ denote two distributions. These distributions share the same shape if constants $a>0$ and $b$ exist such that $Y=a(X-b)$.

(Manuscript received May 27, 2009; revision accepted for publication May 13, 2010.) 